Methods Community consultative processes were used to engage First Nations, Inuit and Métis (FN/I/M) youth in the development of sexual health promotion and STBBI prevention approaches. Engagement began in 2008 through the establishment of a steering committee, which included FN/I/M youth stakeholders, with representatives from governmental and non-governmental agencies. The steering committee aimed to make effective use of relevant technologies and social media to reach and engage FN/I/M youth in Canada. In 2009, a workshop was held to enhance the involvement of youth to identify priorities for STBBI messaging and explore the best mediums to reach youth. The outcomes of this consultation process informed the development of capacity building social media pilot projects to reach $\mathrm{FN} / \mathrm{I} / \mathrm{M}$ youth.

Results FN/I/M youth identified the importance of taking a holistic approach by viewing sexual health through the lens of social determinants. Youth highlighted poverty, social support networks and education as major influences on sexual health outcomes for their peers. Key challenges youth noted included the lack of sexual health education and the need to strengthen social support networks. By tying in these determinants into promotion, youth identified themes such as addressing homophobia, and raising the importance of cultural relevancy to de-stigmatise sexual health within FN/I/M communities and to help youth to relate closer to messages. Youth also indicated that peer-to-peer social media methods were an ideal mechanism to convey messages. Many youth from across Canada were engaged in developing their own STBBI messages.

Conclusions Engaging FN/I/M youth as partners from concept onwards has led to an informed approach to the development of sexual health promotion and STBBI prevention messaging. Public health practitioners learnt youth need to be involved to gain credibility within this population, youth capacity building is important and taking a holistic social determinants approach in conceptualising messages is critical when reaching FN/I/M youth.

\section{2-S2.06 REDUCING SEXUAL RISK BEHAVIOUR AMONG YOUTH: THE DEVELOPMENT AND EFFECT EVALUATION OF AN INTERACTIVE ONLINE INTERVENTION FOR INDIVIDUALS AND THEIR SEXUAL NETWORK}

doi:10.1136/sextrans-2011-050109.78

${ }^{1} \mathrm{U}$ Davidovich, ${ }^{2} \mathrm{H}$ Uhr-Dall. ${ }^{1}$ Amsterdam Public Health Service, Amsterdam, Netherlands; ${ }^{2}$ Amsterdam Public Health Service, Netherlands

Background This study presents an intervention and its evaluation of an innovative approach to counteract barriers to safe sex and STI testing of youth aged $16-24$.

Methods The intervention-http://www.vrijlekker.nl/- has been developed based on over 200 qualitative interviews with youth investigating barriers to safe sex and STI testing. Based on these interviews, training modules were developed which participants could follow online. These modules aimed at counteracting the individual barriers for safe sex and STI testing as well as removing impeding elements in the social and sexual network. The Information-, Motivation and Behavioural Skills (IMB) model (Fisher \& Fisher) was used as the theoretical basis of the intervention. The training included filmed coaches that guided users throughout the intervention, thematic films, interactive text with personal feedback and sexual network tools. The modules were offered on a tailored basis to match each user's own cognitive \& behavioural risk profile. This profile was established via an automated online intake. An evaluation was conducted comparing a demographically matched control group recruited prior to the launch of the intervention and an intervention group. Behavioural outcomes were compared at 6 month follow-up.
Results The evaluation included 2944 participants of whom 1553 completed the follow-up (mean age 19, SD 2.4). The intervention group used condoms significantly more often with their most recent casual partner $[\mathrm{OR}=1.8295 \% \mathrm{CI} 1.08 \%$ to $3.04 \%$ ] and/or with their steady partner $[\mathrm{OR}=2.1795 \% \mathrm{CI} 1.48 \%$ to $3.18 \%$ ] than the control group at 6 months follow-up. Over 170000 unique persons used the intervention already during its first year, and more than 100 new users continue to do so every day. Some schools in the Netherlands have adopted the site as part of their routine sexual education program.

Conclusions This study has shown than offering youth an empirically and theoretically sound intervention that is interactive and which adapts itself to the individual needs of each user and its sexual network, results in a desired behavioural change and high uptake, even among the challenging target group of youth.

\section{Social and behavioural aspects of prevention oral session 3-Sexual and diagnostic behaviours: issues in measurement 02-S3.01 FEASIBILITY AND ACCEPTABILITY OF SELF-SAMPLING
FOR RECTAL SEXUALYY TRANSMITTED INFECTIONS (STI)
AMONG BISEXUAL MEN IN THE USA}

doi:10.1136/sextrans-2011-050109.79

${ }^{1} \mathrm{~B}$ Dodge, ${ }^{1} \mathrm{~B}$ Van Der Pol, ${ }^{1} \mathrm{M}$ Reece, ${ }^{2} \mathrm{D}$ Malebranche, ${ }^{1} \mathrm{D}$ Fortenberry. ${ }^{1}$ Indiana University, Bloomington, USA; ${ }^{2}$ Emory University, Atlanta, USA

Introduction Rectal sexually transmitted infections (STI) are a common health concern for men who have sex with men (MSM). Studies have not yet determined the relevance of these pathogens among men who have sex with both men and women (MSMW). Screening for rectal STI is not currently a widespread option for bisexual men in the USA.

Methods Qualitative data and self-obtained rectal specimens were collected from a diverse sample of bisexual men. Upon completion of the rectal self-sampling, each participant provided information regarding their overall experience with the process.

Results From a total sample of 75 bisexual men, 58 participants provided self-obtained rectal samples. While most men did not test positive, a prevalence $(10.3 \%)$ of C. trachomoatis infection was found in this sample. Men who collected samples reported overall acceptability and comfort with self-sampling for rectal STI. Privacy was a primary concern for men regarding self-sampling. Of the men who did not provide a rectal self-sample, the most common reason was having been tested in the recent past. Discussion: Self-sampling is a feasible and acceptable option when offered to bisexual men. Research and interventions are needed to ascertain which combinations of STI testing (including self-sampling) and treatment methods are most appropriate for diverse groups of bisexual men.

\section{2-S3.02 ASSESSING THE VALIDITY OF SEXUAL BEHAVIOUR REPORTS IN A WHOLE POPULATION SURVEY IN RURAL MALAWI}

doi:10.1136/sextrans-2011-050109.80

${ }^{1} \mathrm{~J}$ Glynn, ${ }^{2} \mathrm{~N}$ Kayuni, ${ }^{2} \mathrm{E}$ Banda, ${ }^{1} \mathrm{~F}$ Parrott, ${ }^{1} \mathrm{~S}$ Floyd, ${ }^{3} \mathrm{C}$ Tanton, ${ }^{1} \mathrm{~J}$ Hemmings, ${ }^{1}$ A Molesworth, ${ }^{1} \mathrm{~A}$ Crampin, ${ }^{1} \mathrm{~N}$ French. ${ }^{1}$ London School of Hygiene \& Tropical Medicine, London, UK; ${ }^{2}$ Karonga Prevention Study, Malawi; ${ }^{3}$ University College London, UK

Background Sexual behaviour surveys are widely used, but underreporting of particular risk behaviours is common, especially by 
women. Surveys in whole populations provide an unusual opportunity to understand the extent and nature of such under-reporting. Methods All consenting individuals aged between 15 and 59 within a demographic surveillance site in northern Malawi were interviewed about their sexual behaviour. Validity of responses was assessed by analysis of probing questions; by comparison of results with in-depth interviews and with Herpes simplex type-2 (HSV-2) seropositivity; by comparing reports to same sex and opposite sex interviewers; and by quantifying the partnerships within the local community reported by men and by women, adjusted for response rates.

Results 6796 women and 5253 men ( $83 \%$ and $72 \%$ of those eligible) consented and took part in sexual behaviour interviews. Probing questions and HSV-2 antibody tests in those who denied sexual activity identified under-reporting for both men and women. Reports varied little by sex or age of the interviewer. The number of marital partnerships reported was comparable for men and women, but men reported about four times as many non-marital partnerships. The discrepancy in reporting of non-marital partnerships was most marked for married women (men reported about seven times as many non-marital partnerships with married women, as were reported by married women themselves), but was only apparent in younger married women see Abstract O2-S3.02 figure 1.

Conclusions We have shown that the under-reporting of non-marital partnerships by women was strongly age-dependent. The extent of under-reporting of sexual activity by young men was surprisingly high. The results emphasise the importance of triangulation, including biomarkers, and the advantages of considering a whole population.

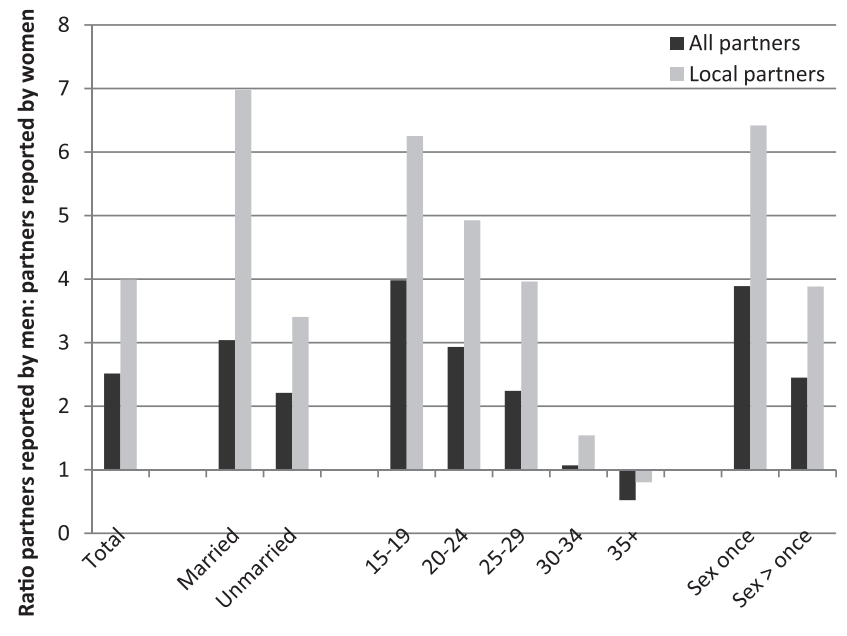

Characteristics of female partner and partnership

Abstract 02-S3.02 Figure 1 Ratio of non-marital partners reported by men compared to women, by characteristics of the women and partnership

\section{2-S3.03 DURATION OF RECENT SEXUAL PARTNERSHIPS IN SEXUALLY ACTIVE MEN AND WOMEN}

doi:10.1136/sextrans-2011-050109.81

Jami Leichliter, Sevgi Aral. Centers for Disease Control \& Prevention, Atlanta, USA

Background The duration of partnerships and partner change rates contribute to STI transmission. Additionally, concurrent partnerships and short gaps between partnerships may be associated with STI spread. Few studies have examined the duration of sexual partnerships at the national level. We examined the duration of recent sexual partnerships reported by sexually active men and women in the US.
Methods Data were from a national survey of reproductive age men and women in the US 7470 men and women reported having an opposite-sex partner in the past year and reported on their recent sex partners (up to three). Duration of partnerships was measured in months; partnerships where respondents reported only having sex once were coded as 1-month in duration. Respondents indicated whether or not the partner was a current sex partner. Bivariate analyses examined duration of partnerships (mean) by demographics. Data were analysed separately for men and women. A subset of unmarried respondents was also examined.

Results Findings were similar for the entire sample and for those who were unmarried. Duration of most recent partnership was longer for women as compared to men, but men reported longer 2 nd and 3rd most recent partnerships than women. Adolescents had the shortest duration for all three partnerships and were least likely to report that the partner was a current sex partner. There was no difference in duration of partnerships by race with the exception of black men having a shorter duration most recent partnership than other men. More black men but fewer black women reported that their most recent partner was a current partner as compared to other groups. However, black and Hispanic men were more likely to indicate that their second recent partnership was also current. For most recent and 2 nd recent partnerships, married men and women reported the longest duration. For most recent partnership, unmarried men and all women with less than a high school education had a longer duration than their counterparts. There were no differences in whether the partner was current by education.

Conclusions Adolescents and black men had the shortest partnerships. Black women did not differ from white or Hispanic women in the duration of their partnerships. Men and women with short partnerships, overlapping and non-overlapping, may play a key role in the transmission of STIs.

\section{2-S3.04 WEB-BASED SEX DIARIES AND YOUNG MEN WHO HAVE SEX WITH MEN: ASSESSING FEASIBILITY, REACTIVITY, AND DATA AGREEMENT}

doi:10.1136/sextrans-2011-050109.82

S Glick, R Winer, M Golden. University of Washington, Seattle, USA

Background Web-based diaries are a promising means for collecting detailed sexual behaviour and substance use data. Few studies have evaluated the feasibility and reliability of collecting these data among men who have sex with men (MSM). We compared quantitative diary data with retrospective survey data collected over 1year in a cohort of young MSM in Seattle, WA.

Methods Ninety-five MSM, age 16-30, completed web-based surveys every 3 months and were randomised to 1 of 4 diary submission schedules: every 2 weeks, once a week, twice a week, or never. The diaries asked questions about daily sexual behaviour and substance use. We assessed the agreement between diary and survey data using $\kappa$ statistics for dichotomous variables and concordance correlation coefficients (CCC) for continuous variables. We used generalised estimating equations to assess for differences in survey data over time between those assigned an active diary schedule to those assigned no diary.

Results During the first 6 months, $78 \%$ of participants assigned to an active diary schedule completed at least $80 \%$ of their diary days, and the 2-week schedule had the highest and most consistent completion rate. Completion rates dropped to $<60 \%$ during the second 6 months. Among participants with active diary schedules, most measures had strong agreement between the diary and survey data (ie, $\kappa$ or $C C C \geq 0.8$ ). The exceptions to this were numbers of overall and unprotected anal sex acts during the first 3 months ( $C C C=0.53$ and 0.57 , respectively) and alcohol use during the first 